\title{
ON NILPOTENT DERIVATIONS OF PRIME RINGS
}

\author{
CHEN-LIAN CHUANG \\ (Communicated by Donald S. Passman)
}

\begin{abstract}
Let $R$ be a prime ring with center $Z$ and let $U$ be a noncentral Lie ideal of $R$. Suppose that $d$ is a derivation of $R$ such that $d^{n}(u) \in Z$ for all $u \in U$, where $n$ is a fixed integer. It is shown that either $d^{n}(R)=0$ or $R$ is an order of a 4-dimensional simple algebra over a field of characteristic 2 .
\end{abstract}

Let $R$ be a prime ring with center $Z$ and $d$ a derivation of $R$. Chung and J. Luh [4] proved that if $d^{n}(I)=0$ for a nonzero ideal $I$ of $R$, then $d^{n}(R)=$ 0 . The similar result for Lie ideals was also proved in the dissertation of David W. Jensen of North Carolina State University. On the other hand, Lee and Lee [11] generalized Chung and Luh's result by using the condition that $d^{n}(I) \subseteq Z$. For the corresponding generalization on Lie ideals, a partial result has been recently obtained by Trzepizur [15] with the assumption that char $R>2 n$. Our aim here is to prove this generalization in its full generality. The main feature of our argument is, instead of elementwise computation as in previous work, the use of Kharchenko's powerful theorem on differential identities along with structure theorems. This seems to suggest a more unified and effective approach for problems of this sort.

Our main result is the following

Theorem. Let $R$ be a prime ring with center $Z$ and $d$ a derivation of $R$ such that $d^{n}(U) \subseteq Z$, where $U$ is a noncentral Lie ideal of $R$ and $n$ is a fixed natural number. Then $d^{n}(R)=0$ unless $R$ is an order of a 4-dimensional simple algebra over a field of characteristic 2 .

Combining with the result in [1], we immediately obtain the following

Corollary. Let $R$ be a prime ring with center $Z$ and let $p\left(x_{1}, \ldots, x_{m}\right)$ be a noncentral polynomial in noncommuting indeterminates $x_{1}, \ldots, x_{m}$ and with coefficients in the extended centroid $C$. Let $d$ be a derivation of $R$ such that $d^{n}\left(p\left(x_{1}, \ldots, x_{m}\right)\right) \in Z$ for all $x_{1}, \ldots, x_{m}$ in a nonzero ideal $I$ of $R$. Then $d^{n}(R)=0$ unless $R$ is an order of a 4-dimensional simple algebra over a field of characteristic 2 .

Received by the editors September 2, 1987 and, in revised form, January 26, 1989.

1980 Mathematics Subject Classification (1985 Revision). Primary 16A72.

Key words and phrases. Prime rings, Lie ideals, derivations, differential identities. 
We start with the following simple lemma, which is useful for pushing results from ordinary ideals to Lie ideals. Though our formulation below is only for one indeterminate for simplicity, the lemma can be extended easily to more indeterminates.

Lemma 1. Let $D$ be a division ring and let ${ }_{D} V$ be an infinite-dimensional left $D$ vector space. Suppose that $R$ is a dense subring of $\operatorname{Hom}\left({ }_{D} V,{ }_{D} V\right)$ and that $\phi(x)$ is a generalized polynomial with coefficients in $\operatorname{Hom}\left({ }_{D} V,{ }_{D} V\right)$. If $\phi([x, y])=0$ is a GPI of $R$, then $\phi(x)=0$ is a GPI of $\operatorname{Hom}\left({ }_{D} V,{ }_{D} V\right)$.

Proof. Since generalized polynomials are continuous with respect to the finite topology of $\operatorname{Hom}\left({ }_{D} V,{ }_{D} V\right)$ (see [6, p. 248] for the definition), it suffices to show that the set $\{[x, y]: x, y \in R\}$ is dense in $\operatorname{Hom}\left({ }_{D} V,{ }_{D} V\right)$ with respect to the finite topology. So let $a \in \operatorname{Hom}\left({ }_{D} V,{ }_{D} V\right)$ and let $v_{1}, \ldots, v_{n} \in V$ be $D$-independent vectors. We must show that there exist $x, y \in R$ such that $v_{i}[x, y]=v_{i} a$ for $i=1, \ldots, n$. Since $\operatorname{dim}_{D} V=\infty$, there exist $u_{1}, \ldots, u_{n} \in$ $V$ such that $u_{1}, \ldots, u_{n}$ are $D$-independent modulo the $D$-subspace spanned by $v_{1}, \ldots, v_{n}$. By the density of $R$ in $\operatorname{Hom}\left({ }_{D} V,{ }_{D} V\right)$, there exist $x, y \in R$ such that $v_{i} x=u_{i}, v_{i} y=0$ and $u_{i} y=v_{i} a$ for $i=1, \ldots, n$. Now we verify easily that $v_{i}[x, y]=v_{i}(x y-y x)=v_{i} x y=u_{i} y=v_{i} a$ for $i=1, \ldots, n$.

The next lemma treats the case $n=1$ and is well known more or less in the literature. We include here the proof for the sake of completeness.

Lemma 2. When $n=1$, the theorem holds.

Proof. In the notation of the theorem above, $d(U) \subseteq Z$ implies $d([U, U])=$ 0 . By [10], the subring generated by $[U, U]$ contains a nonzero ideal $I$ of $R$, unless $\operatorname{char} R=2$ and $R$ satisfies the standard polynomial identity $S_{4}$, which is excluded by our assumption. So $d(I)=0$ and $d(R)=0$ follows.

Proof of theorem. Assume on the contrary that our theorem is false. Let $n$ be the minimal integer such that there exists a derivation $d$ of a prime ring $R$ with $d^{n}(R) \neq 0$ but with $d^{n}(U) \subseteq Z$ for a noncentral Lie ideal $U$ of $R$. Fix such $R, U$ and $d$. Let $Q$ be the two-sided Martindale quotient ring of $R$. The center $C$ of $Q$ is called the extended centroid of $R$.

By [11], there exists a nonzero ideal $I$ of $R$ such that $U \supseteq[I, R]$. Then the differential identity $\left[d^{n}([x, y]), z\right]=0$ holds on $Q$ by [3, Theorem 1]. Set $W=[Q, Q]$. Then $W$ is a noncentral Lie ideal of $Q$ and $d^{n}(W) \subseteq C$. The argument of the first three claims below is adopted from [11].

Claim 1. $n$ is invertible in $C$. Assume on the contrary that char $R=p>0$ and $p$ divides $n$. Then $\delta=d^{p}$ is also a derivation of $R$ and $\delta^{(n / p)}(U)=$ $d^{n}(U) \subseteq Z$. Since $n / p<n$, this contradicts with the minimality of $n$.

Claim 2. $d^{n}(C)=0$. Assume on the contrary that $d^{n}(\alpha) \neq 0$ for some $\alpha \in C$. Observe that $d^{i}(W) \subseteq W$ for each integer $i \geq 1$. So for $w \in W, \alpha d^{n-1}(w) \in W$ 
and hence $d^{n}\left(\alpha d^{n-1}(w)\right) \in C$. Expanding this,

$$
d^{n}(\alpha) d^{n-1}(w)+\sum_{i=1}^{n}\left(\begin{array}{c}
n \\
i
\end{array}\right) d^{n-i}(\alpha) d^{n+i-1}(w) \in C .
$$

Since each term in the summation is already in $C, d^{n}(\alpha) d^{n-1}(w) \in C$. Hence $d^{n-1}(W) \subseteq C$, a contradiction of the minimality of $n$.

Claim 3. $d(C)=0$. Let $m$ be the minimal integer $\leq n$ such that $d^{m}(C)=0$. Assume on the contrary that $m \geq 2$. Pick $\alpha \in C$ such that $d^{m-1}(\alpha) \neq 0$. For $w \in W$, we have

$$
d^{n}\left(d^{m-2}(\alpha) w\right)=d^{m-2}(\alpha) d^{n}(w)+n d^{m-1}(\alpha) d^{n-1}(w) \in C .
$$

So $n d^{m-1}(\alpha) d^{n-1}(w) \in C$. Since $n$ is invertible in $C$ by Claim $1, d^{n-1}(w) \in$ $C$ for all $w \in W$, a contradiction of the minimality of $n$. So $m=1$ and $d(C)=0$ as desired.

Claim 4. $Q$ satisfies a nontrivial GPI. By means of the basic identities $1-6$ of [8], the differential polynomial $d^{n}(x)$ gives rise to a reduced expression

$$
\phi(x)=\sum_{i} \sum_{j} a_{i j} \Delta_{i}(x) b_{i j},
$$

where $a_{i j}, b_{i j} \in R$ and where $\Delta_{i}$ are distinct regular derivation words. (In the notation employed in [7] and [8], where derivations are put on the upper right corner, we should write $\phi(x)=\sum_{i} \sum_{j} a_{i j} x^{\Delta_{i}} b_{i j}$ instead.) The reduced expression $\phi(x)$ is said to be nontrivial if the corresponding GP $\sum_{i} \sum_{j} a_{i j} z_{i} b_{i j}$, where $z_{i}$ are distinct new indeterminates, is nontrivial. If the reduced expression $\phi(x)$ is trivial, then $\phi(x)=0$ holds trivially on $R$ and hence so does the differential identity $d^{n}(x)=0$. This contradicts with our choice of $n$. So the reduced expression $\phi(x)$ must be nontrivial. By reindexing, we may assume that $\Delta_{1}$ is the largest derivation word such that the reduced expression $\sum_{j} a_{1 j} \Delta_{1}(x) b_{1 j}$ is nontrivial. Write each $\Delta_{i}$ as $\Delta_{i}=\delta_{1}, \ldots, \delta_{m}$. Using Leibniz's rule for derivations, we may expand

$$
\begin{aligned}
\Delta_{i}([x, y]) & =\delta_{1} \cdots \delta_{m}([x, y]) \\
& =\left[\Delta_{i}(x), y\right]+\left[\delta_{1} \cdots \delta_{m-1}(x), \delta_{m}(y)\right]+\cdots .
\end{aligned}
$$

Observe that this expansion also gives rise to a reduced expression and contains exactly one term involving $\Delta_{i}(x)$, namely $\left[\Delta_{i}(x), y\right]$. Hence, the similar expansion of $\phi([x, y])$ is also reduced and the sum $\sum_{j} a_{1 j}\left[\Delta_{1}(x), y\right] b_{1 j}$ consists of all terms involving $\Delta_{1}(x)$. (Since $\Delta_{1}$ is the largest derivation word, the expansion of other $\Delta_{i}([x, y]) \quad(i \neq 1)$ cannot contribute to terms involving $\Delta_{1}(x)$.) Consequently, $\phi([x, y])$ gives rise to a nontrivial reduced expression and hence so does $[\phi([x, y]), z]$. But $[\phi([x, y]), z]=0$ holds on $R$ since so does $\left[d^{n}([x, y]), z\right]=0$. Now applying [7, Corollary $5, \mathrm{p}$. 163] to the reduced expression of $[\phi([x, y]), z], R$ satisfies a nontrivial GPI. (For example, 
we can see that $R$ satisfies the nontrivial GPI $\left[\sum_{j} a_{1 j}[x, y] b_{1 j}, z\right]=0$.) By [2, Theorem 2], $Q$ also satisfies a nontrivial GPI as desired.

Using Claim 3 and Claim 4 , by the result on [8, p. 68], there exists $a \in Q$ such that $d(x)=[a, x]$ for all $x \in R$. Hence $d^{n}(x)$ assumes the simple form $d^{n}(x)=[a, \ldots,[a, x]]$ and is merely an ordinary generalized polynomial.

By [12], $Q$ has a minimal idempotent $e$ such that $D=e Q e$ is a finitedimensional division algebra over $C$. Set $V=e Q$. Then $V$ is a left $D$-vector space. Via right multiplication on $V, Q$ can be realized as a dense subring of $\operatorname{Hom}\left({ }_{D} V,{ }_{D} V\right)$.

If $\operatorname{dim}_{D} V=\infty$, then $d^{n}([x, y])=0$ holds on $Q$ by [14, Theorem 4] and, consequently, $d^{n}(x)=0$ holds on $\operatorname{Hom}\left({ }_{D} V,{ }_{D} V\right)$ by Lemma 1 . But then $d^{n}(R)=0$, a contradiction. So we must assume that $V$ is of finite $D$ dimension. Thus $Q$ is equal to $\operatorname{Hom}\left({ }_{D} V,{ }_{D} V\right)$ and hence is isomorphic to $D_{m}$, the $m \times m$ matrix ring over $D$, for some $m$.

Let $\Phi$ be a maximal separable subfield of $D$ and let $F$ be the algebraic closure of $\Phi$. Then the ring $S=Q \otimes_{C} F$ is isomorphic to the $m \times m$ matrix ring $F_{m}$ for some $m$. The multilinear condition $d^{n}([x, y]) \in C$ for $x, y \in Q$ carries over to $S$ and assumes the form $[a, \ldots,[a,[x, y]]] \in F$ for $x, y \in F_{m}$. Replacing $R$ by $S$, we assume from now on that $R=F_{m}$ for an algebraically closed field $F$ and $d(x)=[a, x]$ for all $x \in F_{m}$.

Since $[a, R] \subseteq[R, R]$, we have that $d^{n+1}(R)=d^{n}([a, R]) \subseteq Z$ and hence $d^{n+2}(R)=0$. Thus $a$ defines a nilpotent derivation on $R$. By [13, Theorem 2] $a-\lambda$ is nilpotent for some $\lambda \in F$. (The assumption on characteristic in [13] is unneeded for nilpotent derivations.) Replacing $a$ by $a-\lambda$, we assume that $a$ is nilpotent. Write $a$ in the Jordan normal form

$$
a=\left(\begin{array}{cccc}
a_{1} & & & 0 \\
& a_{2} & & \\
& & \ddots & \\
0 & & & a_{r}
\end{array}\right)
$$

where $a_{i}$ is the $s_{i} \times s_{i}$ matrix

$$
\left(\begin{array}{cccc}
0 & 1 & \cdots & 0 \\
& 0 & \ddots & \vdots \\
& & \ddots & 1 \\
0 & & & 0
\end{array}\right) \quad(i=1, \ldots, r) .
$$

We may assume that $s_{1} \geq s_{2} \geq \cdots$. Note that $s_{1} \geq 2$, for otherwise, $a=0$.

Let $e_{i j}$ be the matrix of $F_{m}$ with 1 in the $(i, j)$-entry and 0 elsewhere. We claim that $d^{n}\left(e_{21}\right)=0$. Assume towards a contradiction that $d^{n}\left(e_{21}\right) \in F \backslash\{0\}$. By direct computation, $a^{2} e_{21}=0$. Hence $a^{2} d^{n}\left(e_{21}\right)=d^{n}\left(a^{2} e_{21}\right)=0$. So $a^{2}=0$. Using this, we compute directly that $d^{2}(x)=-2 a x a$ and $d^{3}(x)=0$. If $n \geq 3$, then $d^{n}(R)=0$, contradicting our choice of $n$. If $n=2$, then $d^{2}\left(e_{21}\right)=-2 a x a$ cannot be invertible and, as a central element, must be zero. 
So we are left with the case $n=1$, which is also impossible by Lemma 2 . Hence $d^{n}\left(l_{21}\right)=0$ as claimed.

Since $e_{11}=a e_{21}, d^{n}\left(e_{11}\right)=d^{n}\left(a e_{21}\right)=a d^{n}\left(e_{21}\right)=0$. Given $x \in R$, consider, $x_{1}=x-\operatorname{trace}(x) e_{11}$. Then $\operatorname{trace}\left(x_{1}\right)=0$ and hence $x_{1} \in[R, R]$. So $d^{n}\left(x_{1}\right) \in Z$. But $d^{n}\left(x_{1}\right)=d^{n}(x)-\operatorname{trace}(x) d^{n}\left(e_{11}\right)=d^{n}(x)$. We have thus shown that $d^{n}(x) \in Z$ for all $x \in R$. Replacing $x$ by $a x$, we have $d^{n}(a x)=a d^{n}(x) \in Z$, for $x \in R$. If $d^{n}(x) \in Z \backslash\{0\}$ for some $x$, then $a \in Z$, a contradiction again. This completes the proof.

The exceptional case excluded by the theorem above really happens: Let $R$ be the ring of all $2 \times 2$ matrices over a field of characteristic 2 . Let $d$ be the inner derivation defined by $\left(\begin{array}{ll}0 & 1 \\ 0 & 0\end{array}\right)$. We can verify easily that $d([R, R]) \subseteq Z$. But nothing can be said about $d(R)$.

\section{ACKNOWLEDGMENT}

The author would like to express his sincere thanks to the referee for the valuable suggestions which help to clarify and simplify the whole paper. In particular, the proof of Lemma 1 is due to the referee.

\section{REFERENCES}

1. C.-L. Chuang, The additive subgroup generated by a polynomial, Israel J. Math. 59 (1987) (1), 98-106.

2. 723-728.

3. __ On compositions of derivations of prime rings, (to appear in Proc. Amer. Math. Soc.).

4. L. O. Chung and J. Luh, Nilpotency of derivatives on an ideal, Proc. Amer. Math. Soc. 90 (1984) (2), 211-214.

5. I. N. Herstein, A note on derivations II, Canad. Math. Bull. 22 (1979) (4), 509-511.

6. N. Jacobson, Lectures in abstract algebra (II), D. Van Nostrand Company, Inc., New York, 1953.

7. V. K. Kharchenko, Differential identities of prime rings, Algebra i Logika 17 (1978) (2), 220238 (English translation, 154-168).

8. __ Differential identities of semiprime rings, Algebra i Logika 18 (1979) (1), 86-119 (English translation, 58-80).

9. J. Krempa and J. Matczuk, On the composition of derivations, Rend. Circ. Mat. Palermo (2) 33 (1984), 441-455.

10. C. Lanski and S. Montgomery, Lie structure of prime rings of characteristic 2, Pacific J. Math. 42 (1972), 117-136.

11. P. H. Lee and T. K. Lee, Note on nilpotent derivations, Proc. Amer. Math. Soc. 98 (1986) (1), $31-32$.

12. W. S. Martindale III, Prime rings satisfying a generalized polynomial identity, J. Algebra 12 (1969), 574-584.

13. W. S. Martindale III and C. R. Miers, On the iterates of derivations of prime rings, Pacific J. Math. 104 (1983) (1), 179-190.

14. L. H. Rowen, Generalized polynomial identities, J. Algebra 34 (1975), 458-480.

15. A. Trzepizur, Lie ideals of prime rings with derivations of higher order, Bull. Soc. Belg. Sér. B 38 (1986) (2), 225-228.

Department of Mathematics, National Taiman University, Taipei, Taiwan 10764, REPUBLIC OF CHINA 